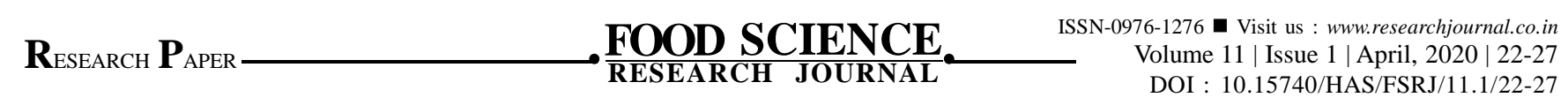

\title{
Development of mango extrudates by extrusion processing
}

\author{
Dhruv Juyal
}

Fruits and vegetables are an important nutritional requirement for human beings as these foods not only meet the quantitative needs to some extent but also supply vitamins and minerals, which improve quantity of our diet and maintain health. It is, therefore, necessary to make them available for consumption throughout the year in processed form. Drying is the oldest method for preserving food as it controls microbial growth, reduces size of food, thus, providing it good storage and packaging properties. Fruits and vegetables selected for drying should be of the best quality- fresh and ripe. To dry them successfully these should have good rheological and textural properties. The good quality fresh mangoes were procured from the market, washed, peeled and processed to get the pulp. The mango kurkure made from roasted wheat and barley was uniform in texture, lacked crispiness and gave good flavour.

Key Words : Mango extrudate, Flavour, Texture, Extruder, Mango pulp

How to cite this article : Juyal, Dhruv (2020). Development of mango extrudates by extrusion processing. Food Sci. Res. J., 11(1): 22-27, DOI : 10.15740/HAS/FSRJ/11.1/22-27.Copyright@ 2020: Hind Agri-Horticultural Society. 